\title{
SELECTION OF SWITCHING SITES IN ALL-OPTICAL NETWORK TOPOLOGY DESIGN
}

\author{
Shivashis Saha ${ }^{\dagger}$, Eric D. Manley ${ }^{\star}$, Jitender S. Deogun ${ }^{\dagger}$ \\ ${ }^{\dagger}$ Department of Computer Science and Engineering, University of Nebraska-Lincoln, Lincoln, NE 68588-0115, U.S.A. \\ ${ }^{\star}$ Department of Mathematics and Computer Science, Drake University, Des Moines, IA 50311, U.S.A. \\ Email: ssaha@cse.unl.edu, eric.manley@drake.edu,deogun@cse.unl.edu
}

\begin{abstract}
In this paper, we consider the problem of topology design for both unprotected and one-link protected all-optical networks. We investigate the problem of selecting switching sites to minimize total cost of the network. The cost of an optical network is expressed as a sum of three main factors: the site cost, the link cost, and the switch cost.

For unprotected networks with linear cost model, we present a mixed integer linear programming (MILP) formulation of the problem. We also present an efficient heuristic to approximate the solution. The experimental results show good performance of the linear cost model heuristic. In $16 \%$ of the experiments with 10 nodes network topologies, the linear cost model heuristic had no error. Moreover, for $54 \%$ and $86 \%$ of the experiments with 10 nodes network topologies, the linear cost model heuristic's solution is within $2 \%$ and $5 \%$ of its optimal value respectively.

Finally, we extend our approach to one-link protected networks, and present an efficient survivable heuristic, and representative experimental results.
\end{abstract}

\section{KEY WORDS}

Optical Networks, Network Optimization, Switching Site, Mixed Integer Linear Programming

\section{Introduction}

The need for eliminating costly $\mathrm{O} / \mathrm{E} / \mathrm{O}$ conversions to reduce the mismatch between the capacity and speed of the optical and electronic transmissions motivates the design of an all-optical network. Switching complexity plays a significant role in the cost of an optical switch. Two types of cost models for switch cost has been proposed in the literature [1], [2], [3]. First, the quadratic cost model in which the cost of an optical switch increases quadratically with the increase in the number of input signals [2]. Second, the linear cost model in which the cost of an optical switch increases linearly with the increase in the number of input signals [3]. Optical switches based on ATM equipments [1] mostly follow quadratic model [2]. For example, $2 \times 2$ and $8 \times 8$ optical switches may cost about $\$ 100$ and $\$ 2,000$ respectively, whereas $1024 \times 1024$ optical switches may cost as much as $\$ 4,000,000$ [2]. Optical switches based on ROADM and WSS technologies have linear cost model [3].
Several other cost effective designs of all-optical interconnects have also been proposed in [4] and [5]. The designs of all-optical interconnects proposed in [4] perform switching as a combination of wavelength and fiber crossconnect. As these switches are not yet realized, a cost model for these kind of switches is not known [4].

In order to design a minimum cost all-optical network topology, we need to minimize the sum of three main factors: the site cost, the link cost, and the switch cost. The optical crossconnect site (simply, site) cost includes the installation, real estate, and maintenance costs of the optical switch at the site. The site cost varies from one site to another and is also influenced by its geographic location. The cost of an optical link between two sites is generally proportional to the distance between them [6]. The unit cost of a link may also vary depending on its geographic location and the cost of laying or leasing optical fiber [6]. According to a recent study, the cost of laying fiber is $\$ 200,000$ $\$ 300,000$ per mile [7]. However, the ratio of switch cost to the link cost varies significantly. On one hand, with increasing switching complexity and the number of wavelengths in a fiber, the cost of an optical switch is increasing significantly [6]. On the other hand, in large scale networks, the switch cost may be significantly smaller when compared to the link cost [7]. All these factors need to be addressed when minimizing the total network cost.

In [8], we presented preliminary results for this problem with a specialized cost model for unprotected networks. We extend this work in this paper ${ }^{1}$ by presenting a heuristic and a mixed integer linear programming (MILP) formulation of the problem with linear cost model for unprotected networks. We perform extensive experiments with different parameter settings to evaluate the performance of the heuristic. We also present a more thorough evaluation using our previously presented quadratic cost model. In our experiments, a commercial optimizer could not solve the the MILP and MIQP formulations even with 20 nodes in a reasonable amount of time. Finally, we extend our approach to one-link protected networks.

\footnotetext{
${ }^{1}$ Preliminary versions of this work appeared in IEEE ANTS 2009 [8] and in an unpublished technical report TR-UNL-CSE-2009-114 [11]
} 


\section{Related Work}

A survey of models and optimization methods for designing survivable networks is presented in [9]. The identification of switching sites and the design of physical topology are considered as two independent problems in these models. The authors have studied the trade off between fibers and wavelength leading to a cost effective transport network design.

The minimum number of switching sites required in a network topology for total connectivity can be estimated using the heuristic presented in [10]. The heuristic finds a maximum-leaf spanning tree of the given network topology for minimizing the number of switching sites.

The importance of jointly optimizing the switching and fiber link costs was initially presented in [6]. Two heuristic algorithms are investigated that minimize the total network cost for backbone-protected and unprotected topologies. The heuristic algorithm for minimum total network cost of unprotected networks finds a minimum weight spanning tree among all the nodes identified as a switching site using the heuristic in [10]. The remaining nodes are identified as non-switching sites and are connected to one of the closest switching sites. Similarly, the heuristic for minimum total network cost of backbone-protected networks finds a simple cycle connecting all nodes identified as a switching sites using the heuristic in [10]. The heuristic also ensures that each non-switching site has at least one neighbor which is a switching site.

The network model in [6] and [10] assumes all sites in the network are equally favorable for selection as a switching site. The optical link cost is considered as the Euclidean distance between the sites satisfying the triangle inequality.

We addressed the problem of minimizing the total network cost in [8] and [11]. We presented an MIQP formulation for the quadratic cost model. This quadratic problem formulation is applicable when ATM switches are used in the network. We also presented an effective heuristic to approximate the solution. However, with the advancement in photonic and WDM technologies, optical switches based on ROADM and WSS technologies have linear cost model [3]. Thus, the quadratic problem formulation may not be suitable for the networks having switches with ROADM and WSS technologies.

In this paper, we present an approach for designing minimum cost topologies with both unprotected and onelink protected scenarios. In our approach, the total network cost is expressed as a sum of the costs of the site, link, and switch. The problem for unprotected networks can be formulated as a generalization of the maximum-leaf spanning tree problem, which is $\mathcal{N} \mathcal{P}$-hard [12]. For unprotected networks, we present a heuristic and an MILP formulation for the linear cost model, perform extensive experiments for both linear and quadratic formulations, and analyze the performance of both the heuristics. Sections 3 to 5 are devoted to unprotected networks. In section 6 we extend our approach to one-link protected networks. The one-link protected problem is $\mathcal{N P}$-hard because it degenerates into Hamiltonian Cycle problem [12]. We also present its problem formulation, an efficient survivable heuristic, and partial experimental results.

\section{Problem Formulation}

In this section, we present an MILP formulation of the problem for unprotected networks with linear cost model, which closely follows the MIQP formulation given in [8].

An optical network topology is represented as a finite, simple, undirected, connected graph $G=(V, E)$. Thus, in the network, $V$ represents the set of nodes (potential switching sites), and $E$ represents the set of edges (potential links). Site Cost, denoted by $\Delta_{i}$ is the cost of switching capability at site $i, i=1$ to $|V|$. This cost includes the installation, real estate, and maintenance costs of an optical switch along with the cost of add/drop multiplexers at that node. Edge Cost, denoted by $C_{i j}$ is the cost of the link $i, j$ (pair of fibers, one in each direction), $i, j \in V$ and $(i, j) \in E$. The cost of laying and/or leasing fiber along with the cost of optical amplifiers and repeaters is also included in the edge cost.

As mentioned earlier, modern optical switches follow linear cost model [3]. The degree of a node, defined as the number of input fiber ports [3] in the minimum cost network topology is denoted by $d_{i}, i=1$ to $|V|$. If each fiber caries a fixed number of wavelengths $W$, then $W d_{i} \times W d_{i}$ optical switch is required at a switching site $i$, where $W d_{i}$ is the number of input signals at node $i$. The complexity and size of an optical switch increases not only with the increase in the number of input fiber ports, but also with the increase in the number of wavelengths in a fiber. Let $\chi$ a constant, represents the rate of increase in the cost of a switch as the size of the switch increases. Thus, minimizing $\chi \sum_{i \in V} d_{i}$ minimizes the size of the optical switch and thus minimizes the total switch cost.

\subsection{Formal Problem Statement}

\section{Given:}

- $G=(V, E)$ : A finite, simple, undirected, connected graph representing an optical network topology.

- $C_{i j}$ : The cost the $i, j$ link, $\forall i, j \in V$ and $(i, j) \in E$.

- $\Delta_{i}$ : The site cost of the node $i, \forall i \in V$.

- $\chi$ : The proportionality constant between the cost and size of an optical switch.

Minimize:

$$
\sum_{(i, j) \in E} C_{i j} X_{i j}+\sum_{i \in V} \Delta_{i} S_{i}+\chi \sum_{i \in V} d_{i}
$$

Subject to: 


$$
\begin{aligned}
& X_{i j} \geq X_{i j}^{\prime}, \quad \underset{\quad(i, j) \in E}{ }
\end{aligned}
$$

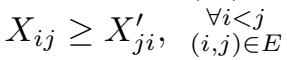

$$
\begin{aligned}
& X_{i j}^{\prime} \geq X_{i j}^{s d}, \quad \begin{array}{c}
\forall s, d \in V \times V \\
s \neq d \\
(i, j) \in E
\end{array} \\
& \sum_{i:(i, j) \in E} X_{i j}^{s d}-\sum_{k:(j, k) \in E} X_{j k}^{s d}=\left\{\begin{array}{ll}
1 & \text { if } j=d \\
-1 & \text { if } j=s \\
0 & \text { otherwise }
\end{array} \quad \forall s, d, j \in V\right. \\
& d_{i}=\sum_{\substack{j: j<i \\
(i, j) \in E}} X_{j i}+\sum_{\substack{k: i<k \\
(i, k) \in E}} X_{i k} \forall i \in V \\
& S_{i} \geq X_{j i}+X_{k i}-1 \forall j, k \text { such that, } \underbrace{}_{\substack{(i, j),(i, k) \in E \\
j \neq k \\
j, k<i}}
\end{aligned}
$$

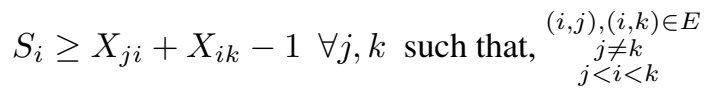

$$
\begin{aligned}
& S_{i} \geq X_{i j}+X_{i k}-1 \forall j, k \text { such that, } \underset{\substack{(i, j),(i, k) \in E \\
j \neq k \\
j, k>i}}{k=k}
\end{aligned}
$$

The first, second, and third terms of the objective function respectively minimize the link, site, and switching costs of a network topology. The minimum network cost topology is a spanning tree of the given network topology [10]. The undirected topology is converted to a directed graph in order to find the spanning tree using $X_{i j}$ and $X_{i j}^{\prime}$ respectively. $X_{i j}^{s d}$ is used to specify a directed path from $s$ to $d$ using link $(i, j) . S_{i}$ identifies sites having more than one neighbors in the spanning tree, which are switching sites.

The given undirected topology is first converted to a directed topology using equations (1) and (2) to find the spanning tree in polynomial time. The equations (3) and (4) intend to find a simple path between any pair of nodes, generating a spanning tree of minimum cost. Equation (5) calculates the degree of a node in the spanning tree. In the spanning tree all non-terminal nodes are identified as switching sites using equations (6), (7), and (8).

When all $\Delta_{i}$ are equal, $C_{i j}=0,(i, j) \in E, i, j \in V$, and $\chi=0$, the problem degenerates to the maximumleaf spanning tree problem [10], which is $\mathcal{N} \mathcal{P}$-hard [12]. Thus, this generalization is also $\mathcal{N} \mathcal{P}$-hard.

The site cost for non-switching sites is not considered in the problem formulation, since it may not have a significant impact on the topology design. However, this cost can be easily incorporated in the problem formulation by introducing an extra term in the problem statement. We assume single fiber per link formulation, but it can be easily extended to solve the multiple fibers per link using a multigraph. The link among several available links between two nodes with least link cost is chosen in the minimum cost network topology. The remaining links are used as backups in the event of failure or regular maintenance. The link with second least link cost is chosen as the primary backup link. Similarly, secondary and tertiary backup paths can also be identified in the network topology, if links exists. For simplicity, we assume a uniform traffic model. Thus, the traffic pattern does not affect the choice of switching sites and optical links.

\section{Proposed Heuristic Approach}

The idea of the proposed linear cost model heuristic for unprotected networks is similar to the heuristic in [8] and [10]. The minimum network cost topology is a spanning tree of the given network topology [10]. For total connectivity in the spanning tree, the switching sites must form a connected topology. Thus, any switching site can communicate with any other switching site in the network topology. Each non-switching site must have exactly one switching site neighbor to have connectivity among all the nodes in the network. Thus, the leaf nodes of the spanning tree are non-switching sites and the non-leaf are switching. It follows that all intermediate nodes on a path between any pair of source and destination nodes must be switching nodes.

A node is said to be covered if it is adjacent to a switching site. Initially, all nodes are non-switching and uncovered. The site with least site cost per uncovered neighbor among all the nodes in the network topology is selected as the first switching site. In subsequent iterations, a new switching site is selected among all covered nonswitching nodes which has the least site cost per neighbor based on previously uncovered neighbors of the selected site. This newly selected switching site is adjacent to one of the old switching sites such that sum of the link cost and the cost involved in increasing the size of the optical switch at the old switching site is minimum among all the possible choices. This greedy selection of switching sites followed by greedy selection of links between them builds a spanning tree, such that the switching sites form a connected topology. The selection of switching sites terminates when all the nodes in the network topology are covered. The leaf nodes of the spanning tree are non-switching sites and the non-leaf are switching. A link between a non-switching site and a switching site is chosen such that the sum of the link cost and the cost of increasing the size of the optical switch at the switching site is minimum among all the possible choices.

\section{- Notations:}

1. $N(i)$ : Number of neighbors of node $i$.

2. $N^{U C}(i)$ : Number of uncovered neighbors of node $i$.

The pseudo-code of the proposed linear cost model heuristic for unprotected networks is given in Figure 1. Its time complexity is dominated by the execution time of steps 3 to 6 . The neighborhood of a vertex can be calculated in $O(E)$ time, if the graph is represented as an adjacency list. Thus, the time complexity of the proposed linear cost model heuristic is $O\left(V^{2} E\right)$. In this paper, we refer to the heuristic for linear (quadratic) cost model as linear (quadratic) heuristic. In section 6, we present the extension to one-link protected networks. 
1. Initialize all nodes to be non-switching and uncovered. Initialize $d_{i}=0, \forall i=1$ to $|V|$.

2. Select the vertex with lowest value of $\frac{\Delta_{i}}{N(i)}, i=1$ to $|V|$, as the first switching site. In case of a tie, choose the node with lowest $\Delta_{i}$.

3. Repeat Steps 4 to 6 until all nodes are covered.

4. Select the node $v$ among all covered nodes which has lowest value of $\frac{\Delta_{i}}{N^{U C}(i)}$ as a switching site, $i=1$ to $|V|, i$ not a switching site, and $N^{U C}(i)>0$.

In case of a tie, choose the node having lowest $\Delta_{i}$.

5. Select a link $(v, x)$, such that $x$ is a switching node and $C_{v x}+\chi * d_{x}$ has lowest value among all choices of $x$. Ties can be broken arbitrarily.

6. Increment the values of $d_{v}$ and $d_{x}$ by 1 .

7. Identify the leaf nodes of the spanning tree as nonswitching sites and the non-leaf as switching.

8. For each non-switching site $y$, select a link $(y, z)$, such that $z$ is a switching site, and $C_{y z}+\chi * d_{z}$ has lowest value among all choices of $z$. Increment the value of $d_{z}$ by 1 . Ties can be broken arbitrarily.

Figure 1. A high-level description of the proposed linear cost model heuristic.

\section{Results and Discussion}

In this section we present the experimental setup, the results from the experiments, and analyze the performance of the linear and quadratic heuristics for unprotected networks.

Experimental Setup: We used the rectangular grid method [13] to generate random connected network topologies for simulation. In this method, nodes are assigned randomly in a rectangular grid. The probability of an edge between two nodes is a function of distance between them [14]. If $d(u, v)$ is the distance between two nodes $u$ and $v, L$ is the maximum distance between any two nodes in the grid, and $\alpha, \beta \in(0,1]$, then the probability of an edge between $u$ and $v$ is:

$$
P(u, v)=\beta^{\frac{-d(u, v)}{L \alpha}}
$$

The edge density of the topology increases with the increase in the value of $\beta$. On the other hand, short edges are more probable than long edges with smaller values of $\alpha$ [14]. We experimented with different combinations of $\alpha$ and $\beta$ and generated three random connected topologies for each of 10, 20, 35, 49, and 86 nodes. We empirically selected the topologies from a set of about 20 candidate network topologies by visual inspection. The random topology generated for 10 nodes using $\alpha=0.5$ and $\beta=0.9$ is shown in Figure 2.

Developing a detailed cost values of the switch, link,

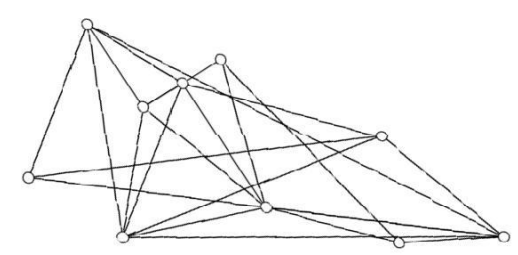

Figure 2. Random topology of 10 nodes $(\alpha=0.5, \beta=$ $0.9)$

and site costs is difficult [3]. Difference in business policies of different vendors is one of the reasons of this difficulty [3]. Moreover, the actual values of these parameters may be confidential and may not be publicly available [3]. The use of different technologies can also result in different values of these parameters [3]. In order to perform a meaningful analysis of the linear and quadratic heuristics, we consider different values of the ratio of the site cost to the link cost and the proportionality constant, $\chi$. The value of the proportionality constant depends on several factors as discussed in Section 3. $\chi$ varies not only with the switch technology, but also with the manufacturer. We believe that the following eight different scenarios lead to an extensive and meaningful analysis: site cost $>>$ link cost, site cost slightly $>$ link cost, site cost $\cong$ link cost, link cost $>>$ site cost, link cost slightly $>$ site cost, and $\chi$ having high, medium, and low values.

The high, medium, and low values of the link and site costs are chosen as a random number between 200 to 250,50 to 100 , and 20 to 60 units respectively. The high, medium, and low values of $\chi$ are chosen as 30,15 , and 5 respectively.

For gaining insight into the impact of different network topologies on the total cost of the network, we generated 81 different network topologies for each network size of $10,20,35,49$, and 86 nodes. The 81 different topologies for each size were generated by choosing 81 combinations of different values of $\alpha, \beta, \chi$, site cost, and link cost. However, due to limited space, only a reasonable sample is shown here.

The experiments were performed on an Intel Core 2 Duo 3.00 Ghz PC with $4 \mathrm{~GB}$ of RAM running Vista. A commercial optimization software, MOSEK [15], has been used to solve the MILP and MIQP formulations [8].

Comparison of Results: Comparison of some the results (45/81) obtained by MOSEK and the proposed linear heuristic for different random topologies of 10 nodes are given in Tables 1, 2 and 3. Comparison of some of the results (30/81) obtained by MOSEK and the quadratic heuristic [8] for different random topologies of 10 nodes are given in Tables 4, 5 and 6. Some of the comparison results for the quadratic cost model were also presented in [8] and [11].

We do not compare our results with [6] because the heuristic in [6] first determines the minimum number of switching sites for total connectivity in the given network topology using the heuristic in [10]. It then finds a minimum weighted spanning tree among all the switching sites and 
connects each non-switching site to one of the switching sites having least link cost. However, the linear heuristic proposed in this paper and the quadratic heuristic in [8] minimizes the sum of the site, link, and switch costs to obtain a spanning tree of minimum network cost of the given network topology. Therefore, comparison of our heuristics with [6] is not meaningful.

Execution Time: The average execution time of the linear heuristic was less than $0.5 \mathrm{sec}$ as compared to 13447 to 497043 secs needed by the MOSEK to solve the MILP formulation for the 10 nodes topologies. The average execution time of the quadratic heuristic was less than $0.5 \mathrm{sec}$ as compared to 9916 to 472785 secs needed by MOSEK to solve the MIQP formulation for 10 nodes topologies.

Error Percentage: The total network cost calculated by the linear heuristic is within $19.58 \%$ of its optimal value in the experiments with 10 nodes topologies. In $16 \%$ of the experiments with 10 nodes topologies, the linear heuristic had no error. Moreover, for $54 \%$ and $86 \%$ of the experiments with 10 nodes topologies, linear heuristic's solution is within $2 \%$ and $5 \%$ of its optimal value respectively. For experiments with 10 nodes topologies, quadratic heuristic's result is within $20.6 \%$ of its optimal value and for $68 \%$ of those experiments, its solution was within $8 \%$ of its optimal value.

A similar comparison for 20 or more nodes topologies could not be performed, because MOSEK could not solve the MILP or MIQP formulations for 20 nodes topologies even in two weeks, as compared to less than two secs taken by both the heuristics on average to solve their respective problem formulations with 86 nodes topologies. Thus, for 20 or more nodes topologies, we only record the total network cost obtained by both the heuristics and analyze their solutions. We perform, but do not report due to limited space, 81 experiments for each of 20,35, 49, and 86 nodes topologies for each of the linear and quadratic formulations.

Table 1. Comparison of some results (15/27) of the linear cost model for $10 \operatorname{nodes}(\alpha=0.5, \beta=0.9)$

\begin{tabular}{|c|c|c|c|c|c|}
\hline $\begin{array}{c}\text { Site } \\
\text { Cost }\end{array}$ & $\begin{array}{c}\text { Link } \\
\text { Cost }\end{array}$ & $\chi$ & $\begin{array}{c}\text { Optimal Cost } \\
\text { (MOSEK) }\end{array}$ & $\begin{array}{c}\text { Total Cost } \\
\text { (heuristic) }\end{array}$ & $\begin{array}{c}\text { Error } \\
\% \text { age }\end{array}$ \\
\hline \hline $200-250$ & $20-60$ & 5 & 857 & 857 & 0.0 \\
$20-60$ & $50-100$ & 5 & 763 & 763 & 0.0 \\
$20-60$ & $200-250$ & 5 & 2078 & 2078 & 0.0 \\
$200-250$ & $200-250$ & 5 & 2476 & 2476 & 0.0 \\
$20-60$ & $20-60$ & 15 & 657 & 679 & 3.34 \\
$50-100$ & $20-60$ & 15 & 763 & 803 & 5.24 \\
$200-250$ & $50-100$ & 15 & 1344 & 1355 & 0.81 \\
$20-60$ & $200-250$ & 15 & 2258 & 2258 & 0.0 \\
$50-100$ & $200-250$ & 15 & 2380 & 2440 & 2.52 \\
$200-250$ & $200-250$ & 15 & 2656 & 2656 & 0.0 \\
$20-60$ & $20-60$ & 30 & 927 & 949 & 2.37 \\
$200-250$ & $20-60$ & 30 & 1307 & 1322 & 1.14 \\
$20-60$ & $50-100$ & 30 & 1213 & 1227 & 1.15 \\
$20-60$ & $200-250$ & 30 & 2528 & 2528 & 0.0 \\
$200-250$ & $200-250$ & 30 & 2926 & 2926 & 0.0 \\
\hline
\end{tabular}

Performance Analysis: The overall performance of
Table 2. Comparison of some results (15/27) of the linear cost model for $10 \operatorname{nodes}(\alpha=0.6, \beta=1.0)$

\begin{tabular}{|c|c|c|c|c|c|}
\hline $\begin{array}{c}\text { Site } \\
\text { Cost }\end{array}$ & $\begin{array}{c}\text { Link } \\
\text { Cost }\end{array}$ & $\chi$ & $\begin{array}{c}\text { Optimal Cost } \\
\text { (MOSEK) }\end{array}$ & $\begin{array}{c}\text { Total Cost } \\
\text { (heuristic) }\end{array}$ & $\begin{array}{c}\text { Error } \\
\text { \%age }\end{array}$ \\
\hline \hline $200-250$ & $20-60$ & 5 & 1033 & 1068 & 3.38 \\
$20-60$ & $50-100$ & 5 & 778 & 778 & 0.0 \\
$50-100$ & $50-100$ & 5 & 901 & 903 & 0.22 \\
$20-60$ & $200-250$ & 5 & 2132 & 2132 & 0.0 \\
$20-60$ & $20-60$ & 15 & 648 & 671 & 3.54 \\
$20-60$ & $50-100$ & 15 & 958 & 958 & 0.0 \\
$50-100$ & $50-100$ & 15 & 1081 & 1083 & 0.18 \\
$200-250$ & $50-100$ & 15 & 1521 & 1556 & 2.30 \\
$20-60$ & $200-250$ & 15 & 2312 & 2312 & 0.0 \\
$200-250$ & $200-250$ & 15 & 2852 & 2863 & 0.38 \\
$20-60$ & $20-60$ & 30 & 918 & 941 & 2.50 \\
$20-60$ & $50-100$ & 30 & 1228 & 1353 & 10.17 \\
$50-100$ & $50-100$ & 30 & 1351 & 1353 & 0.14 \\
$50-100$ & $200-250$ & 30 & 2582 & 2693 & 4.29 \\
$200-250$ & $200-250$ & 30 & 3122 & 3133 & 0.35 \\
\hline
\end{tabular}

Table 3. Comparison of some results $(15 / 27)$ of the linear cost model for $10 \operatorname{nodes}(\alpha=0.7, \beta=0.8)$

\begin{tabular}{|c|c|c|c|c|c|}
\hline $\begin{array}{c}\text { Site } \\
\text { Cost }\end{array}$ & $\begin{array}{c}\text { Link } \\
\text { Cost }\end{array}$ & $\chi$ & $\begin{array}{c}\text { Optimal Cost } \\
\text { (MOSEK) }\end{array}$ & $\begin{array}{c}\text { Total Cost } \\
\text { (heuristic) }\end{array}$ & $\begin{array}{c}\text { Error } \\
\text { \%age }\end{array}$ \\
\hline \hline $20-60$ & $20-60$ & 5 & 463 & 551 & 19.00 \\
$50-100$ & $50-100$ & 5 & 864 & 869 & 0.57 \\
$200-250$ & $50-100$ & 5 & 1169 & 1174 & 0.42 \\
$20-60$ & $200-250$ & 5 & 2109 & 2113 & 0.18 \\
$20-60$ & $20-60$ & 15 & 623 & 745 & 19.58 \\
$50-100$ & $20-60$ & 15 & 643 & 768 & 19.44 \\
$50-100$ & $50-100$ & 15 & 1044 & 1049 & 0.47 \\
$200-250$ & $50-100$ & 15 & 1349 & 1354 & 0.37 \\
$200-250$ & $200-250$ & 15 & 2630 & 2646 & 0.60 \\
$20-60$ & $20-60$ & 30 & 893 & 1035 & 15.90 \\
$200-250$ & $20-60$ & 30 & 1234 & 1353 & 9.64 \\
$20-60$ & $50-100$ & 30 & 1237 & 1271 & 2.74 \\
$200-250$ & $50-100$ & 30 & 1619 & 1624 & 0.30 \\
$50-100$ & $200-250$ & 30 & 2579 & 2611 & 1.24 \\
$200-250$ & $200-250$ & 30 & 2900 & 2916 & 0.55 \\
\hline
\end{tabular}

the linear heuristic is better than the quadratic heuristic. However, both the heuristics produced close to optimal solutions for all the scenarios considered in the experiments. The relatively higher error percentages in some of the experiments are due to the particular settings of the parameters for those experiments.

The performance of both the heuristics is relatively better for high density topologies. With the increase in the density of the topology, the number of choices in the selection of switching sites and optical links also increases. The number of switching sites required for total connectivity is also less in dense topologies as compared with sparse topologies with same number of nodes. Thus, low density of the topology is one of the reasons for not so good performance of the heuristic in some of the experiments shown in Tables 3 and 6.

Nature of Solution: Our experimental results show that both the heuristics are very effective and are not biased toward any specific kind of solutions, e.g. high-congestion 
Table 4. Comparison of some results (10/27) of the quadratic cost model for $10 \operatorname{nodes}(\alpha=0.6, \beta=1.0)$

\begin{tabular}{|c|c|c|c|c|c|}
\hline $\begin{array}{c}\text { Site } \\
\text { Cost }\end{array}$ & $\begin{array}{c}\text { Link } \\
\text { Cost }\end{array}$ & $\chi$ & $\begin{array}{c}\text { Optimal Cost } \\
\text { (MOSEK) }\end{array}$ & $\begin{array}{c}\text { Total Cost } \\
\text { (heuristic) }\end{array}$ & $\begin{array}{c}\text { Error } \\
\% \text { age }\end{array}$ \\
\hline \hline $50-100$ & $50-100$ & 5 & 874 & 996 & 13.95 \\
$20-60$ & $200-250$ & 5 & 2263 & 2387 & 5.47 \\
$200-250$ & $200-250$ & 5 & 2678 & 2730 & 1.94 \\
$200-250$ & $20-60$ & 15 & 1673 & 1714 & 2.45 \\
$200-250$ & $50-100$ & 15 & 1478 & 1578 & 6.76 \\
$50-100$ & $200-250$ & 15 & 2785 & 2921 & 4.88 \\
$200-250$ & $200-250$ & 15 & 2934 & 3012 & 2.65 \\
$50-100$ & $20-60$ & 30 & 1823 & 1967 & 7.89 \\
$200-250$ & $20-60$ & 30 & 2393 & 2434 & 1.71 \\
$200-250$ & $200-250$ & 30 & 3578 & 3689 & 3.10 \\
\hline
\end{tabular}

Table 5. Comparison of some results (10/27) of the quadratic cost model for $10 \operatorname{nodes}(\alpha=0.5, \beta=0.9)$

\begin{tabular}{|c|c|c|c|c|c|}
\hline $\begin{array}{c}\text { Site } \\
\text { Cost }\end{array}$ & $\begin{array}{c}\text { Link } \\
\text { Cost }\end{array}$ & $\chi$ & $\begin{array}{c}\text { Optimal Cost } \\
\text { (MOSEK) }\end{array}$ & $\begin{array}{c}\text { Total Cost } \\
\text { (heuristic) }\end{array}$ & $\begin{array}{c}\text { Error } \\
\% \text { age }\end{array}$ \\
\hline \hline $20-60$ & $50-100$ & 5 & 931 & 954 & 2.47 \\
$20-60$ & $200-250$ & 5 & 2242 & 2278 & 1.60 \\
$20-60$ & $20-60$ & 15 & 1065 & 1279 & 20.6 \\
$20-60$ & $50-100$ & 15 & 1366 & 1486 & 8.78 \\
$200-250$ & $50-100$ & 15 & 1594 & 1741 & 9.22 \\
$20-60$ & $200-250$ & 15 & 2667 & 2711 & 1.64 \\
$200-250$ & $200-250$ & 15 & 2834 & 2878 & 1.55 \\
$20-60$ & $20-60$ & 30 & 1688 & 1868 & 16.64 \\
$50-100$ & $50-100$ & 30 & 2007 & 2105 & 4.88 \\
$20-60$ & $200-250$ & 30 & 3265 & 3298 & 1.01 \\
\hline
\end{tabular}

(a bus) network with long paths, or network with highdegree switches. It just find near minimum cost networks.

Total Cost vs. Topology Design: We found that dense random topologies with longer links have lower total network cost. Moreover, dense networks with fewer constraints are probably better than sparse networks with more constraints. The effect of longer links on the network cost becomes significant in topologies with a large number of nodes. Network topologies with longer links have lower network cost due to less number of switching sites required for total connectivity. However, this insight about long links is essentially just a small-world-graph phenomenon [16].

The heuristics do not attempt to differentiate the network building cost from the operational and maintenance costs. Such issues can be addressed by appropriate amortization schemes.

\section{Link Protected Networks}

Proposed frameworks for both linear and quadratic cost models can be easily extended for protected optical networks which can survive $k$-link failures, $k \geq 1$. In this section, we briefly describe the extension and present representative results for one-link protected networks with linear cost model.

Problem formulation: The one-link protected problem is
Table 6. Comparison of some results (10/27) of the quadratic cost model for $10 \operatorname{nodes}(\alpha=0.7, \beta=0.8)$

\begin{tabular}{|c|c|c|c|c|c|}
\hline $\begin{array}{c}\text { Site } \\
\text { Cost }\end{array}$ & $\begin{array}{c}\text { Link } \\
\text { Cost }\end{array}$ & $\chi$ & $\begin{array}{c}\text { Optimal Cost } \\
\text { (MOSEK) }\end{array}$ & $\begin{array}{c}\text { Total Cost } \\
\text { (heuristic) }\end{array}$ & $\begin{array}{c}\text { Error } \\
\% \text { age }\end{array}$ \\
\hline \hline $20-60$ & $50-100$ & 5 & 917 & 996 & 8.61 \\
$20-60$ & $200-250$ & 5 & 2268 & 2365 & 4.27 \\
$50-100$ & $200-250$ & 5 & 2345 & 2545 & 8.52 \\
$50-100$ & $20-60$ & 15 & 1150 & 1378 & 19.82 \\
$20-60$ & $50-100$ & 15 & 1367 & 1542 & 12.80 \\
$20-60$ & $200-250$ & 15 & 2707 & 3017 & 11.45 \\
$50-100$ & $200-250$ & 15 & 2978 & 3149 & 5.74 \\
$50-100$ & $20-60$ & 30 & 1775 & 1975 & 13.82 \\
$200-250$ & $50-100$ & 30 & 2317 & 2563 & 10.61 \\
$50-100$ & $200-250$ & 30 & 3578 & 3641 & 1.76 \\
\hline
\end{tabular}

$\mathcal{N} \mathcal{P}$-hard as it degenerates into Hamiltonian Cycle problem [12] and thus the $k$-link protected problem is also $\mathcal{N} \mathcal{P}$-hard. The proposed MILP and MIQP formulations can be easily extended to $k$-link protected networks. Eq. 4 of the respective formulations needs to be modified to ensure existence of $k+1$ link disjoint paths between any pair of nodes, thus generating a $k+1$ edge-connected network. The modified equation is:

$$
\sum_{i:(i, j) \in E} X_{i j}^{s d}-\sum_{k:(j, k) \in E} X_{j k}^{s d}=\left\{\begin{array}{ll}
k+1 & \text { if } j=d \\
-k-1 & \text { if } j=s \\
0 & \text { otherwise }
\end{array} \forall s, d, j \in V\right.
$$

Table 7. one-link protected network with 10 nodes $(\alpha=$ $0.5, \beta=0.9)$

\begin{tabular}{|c|c|c|c|c|c|}
\hline $\begin{array}{c}\text { Site } \\
\text { Cost }\end{array}$ & $\begin{array}{c}\text { Link } \\
\text { Cost }\end{array}$ & $\chi$ & $\begin{array}{c}\text { Optimal Cost } \\
\text { (MOSEK) }\end{array}$ & $\begin{array}{c}\text { Total Cost } \\
\text { (heuristic) }\end{array}$ & $\begin{array}{c}\text { Error } \\
\% \text { age }\end{array}$ \\
\hline \hline $20-60$ & $20-60$ & 5 & 947 & 993 & 4.85 \\
$50-100$ & $20-60$ & 15 & 1279 & 1305 & 2.03 \\
$200-250$ & $20-60$ & 15 & 1463 & 1533 & 4.78 \\
$20-60$ & $200-250$ & 30 & 2819 & 2917 & 3.47 \\
$200-250$ & $200-250$ & 30 & 3599 & 3801 & 5.61 \\
\hline
\end{tabular}

Survivable Linear Heuristic: The survivable linear heuristic for one-link protected networks needs to ensure that the network generated is two edge-connected. This can be easily accomplished by appropriately modifying the linear heuristic of Section 4. The main idea is to select two links (rather than a single link in unprotected case) originating from the newly selected switching site in every iteration, except the first. Thus, a new switching site is selected such that it has at least two neighbors among switching sites already identified, and has minimum site cost among all the possible choices. After the new switching site has been identified, two new links are chosen such that the sum of the cost of these links and the cost of increasing the size of the switches at the other switching sites is minimum among all the possible choices. After the second iteration, if adding two new links results in formation of a $C_{3}$ (cycle of three nodes), then the existing (old) link in the $C_{3}$ can be deleted, since the resulting network will remain two edge-connected and will have lower network cost. Finally for each non-switching site, two links to two switching neighbors are identified. Thus, the resulting 
topology is two edge-connected and can survive single link failure.

Discussion: We generated two edge-connected networks using the parameters described in Section 5. We present some of the results in Table 7. The average execution time of MOSEK was around 496245 secs as compared to 2 secs needed by the survivable heuristic on average to solve the corresponding problems with 10 nodes network topologies.

\section{Conclusion}

We investigate the problem of selection of switching sites for minimizing the total network cost for both unprotected and one-link protected networks. In our model, the network cost is expressed as a sum of site, link, and switch costs.

The problem for unprotected networks is formulated as a generalization of the maximum-leaf spanning tree problem, which is $\mathcal{N} \mathcal{P}$-hard. We present an MILP formulation of the problem for unprotected networks with linear cost model. MOSEK could not even solve the MILP formulations with 20 nodes in a reasonable amount of time. Thus an efficient heuristic for linear cost model is proposed. We also perform extensive simulations for both linear and quadratic heuristics [8] and evaluate their performance.

In our experiments for unprotected network, the execution time of MOSEK to solve the MILP formulations with 10 nodes varied within 13447 to 497043 secs, whereas the linear heuristic takes less than $0.5 \mathrm{sec}$ on average. In $16 \%$ of the experiments for unprotected 10 nodes networks, the linear heuristic had no error. Moreover, for 54\% and $86 \%$ of these experiments, the linear heuristic's solution is within $2 \%$ and $5 \%$ of its optimal value respectively. The execution time of MOSEK to solve the MIQP formulations for unprotected 10 nodes networks varied within 9916 to 472785 secs, whereas the quadratic heuristic takes less than $0.5 \mathrm{sec}$ on average. For experiments with unprotected 10 nodes networks, quadratic heuristic's result vary within $1.01 \%$ to $20.6 \%$ of its optimal value and for $68 \%$ of these experiments, its solution is within $8 \%$ of its optimal value. The average execution time required by both the heuristics to solve their respective unprotected formulations with 86 nodes is less than two secs.

We extend our approach to one-link protected networks, and present a survivable heuristic, and representative experimental results to show the effectiveness of the heuristic.

The practicality of our work is in developing heuristics which can be used by network designers as a design tool. As a future work, it would be interesting to extend the proposed formulations for node protected networks.

\section{Acknowledgments}

The authors would like to thank Dr. Byrav Ramamurthy and anonymous reviewers for their insightful comments and suggestions that improved the paper considerably.

\section{References}

[1] K. Schultz and P. Gulak, "Physical performance limits for shared buffer ATM switches," IEEE Trans. Comm., vol. 45, no. 8, pp. 997-1007, 1997.

[2] Y. Leung, "Lightpath Concentrators for All-Optical Networks," Journal of Lightw. Technol., Vol. 24, no. 9, pp. 3259-3267, Sept. 2006.

[3] M. Gunkel, et al.,"A Cost Model for the WDM Layer," International Conference on Photonics in Switching (PS) 2006.

[4] H. S. Hamza and J. S. Deogun, "WDM Optical Interconnects: A Balanced Design Approach,” IEEE/ACM Trans. Networking, vol. 15, no. 6, pp. 1565-1578, Dec. 2007.

[5] Y. Yang and J. Wang, "Designing WDM Optical Interconnects with Full Connectivity by Using Limited Wavelength Conversion," IEEE Trans. Comp., Vol. 53, pp. 1547-1556, 2006.

[6] B. K. Chen and F. A. Tobagi, "Network Topology Design to Optimize Link and Switching Costs", IEEE ICC, pp.2450-2456, June 2007.

[7] Verizon presentation at ECOC 2009.

[8] S. Saha, E. Manley and J. S. Deogun, "Minimizing Network Cost in All-Optical Networks", $3^{\text {rd }}$ IEEE ANTS, pp.1-3, Dec. 2009.

[9] H. Kerivin and A. R. Mahjoub, "Design of survivable networks: A survey", Networks, vol. 46, no. 1, pp 1-21, June 2005.

[10] B. Chen and F. Tobagi, "Optical network design to minimize switching and transceiver equipment costs", BROADNETS, pp.1-10, 2006.

[11] S. Saha, E. Manley and J. S. Deogun, "Selection of Switching Sites in All Optical Network Topology Design”, TR-UNL-CSE-2009-114.

[12] M. R. Garey and D. S. Johnson, "Computers and Intractability: A Guide to the Theory of $\mathcal{N P}$-Completeness", W. H. Freeman, 1979.

[13] B. M. Waxman, "Routing of multipoint connections", IEEE J. Sel. Areas Comm., vol. 6, pp. 1617-1622, Dec. 1988.

[14] Wan-Yi Lin and S. Tjoa, "Network Topology Generators", ENEE698A Communications Seminar, University of Maryland, May 2006.

[15] MOSEK Optimization Software, http://www.mosek.com/

[16] M. Buchanan, "Nexus: Small Worlds and the Groundbreaking Theory of Networks", W. W. Norton \& Company, 2002. 